The first step in defining a physiotherapy plan is to identify if physiotherapy is indicated for that patient. Initial assessment is the first stage and includes a history and thorough examination of the musculoskeletal system to identify any problems the patient may have. Based on these findings treatment goals (short-term and long-term) are determined based on the abilities of the patient and the expectations of the veterinary surgeon and owner. At this point a treatment plan can be established. Where possible, objective outcome measures should be used to monitor progress and, depending on the practice, the veterinary physiotherapist, the veterinary surgeon and the veterinary nurse take part in the discussion.

\section{PHYSIOTHERAPY TREATMENTS}

A wide variety of techniques and modalities are available. The selection of the appropriate treatment method for the individual patient is based on the diagnosis and the initial assessment and will be discussed during the lecture. Often a combination of multiple techniques is applied.

\section{KEY LEARNING OBJECTIVES}

- Understand the key terms used in physiotherapy

- Be able to convey the key benefits of physiotherapy to clients and colleagues
- Understand the indications and contraindications of physiotherapy

\section{MULTIPLE CHOICE QUESTIONS}

1. Physiotherapy may be indicated in which one of the following?
(A) Relaxation
(B) Postoperative rehabilitation or conservative management
(C) Obesity
(D) Behavioural management

2. Physiotherapy aims to achieve which one of the following?

(A) Improved joint and limb function

(B) Improved skin condition

(C) Decreased joint range of motion

(D) Slow down postoperative recovery

3. The first step in defining a physiotherapy plan is:

(A) Assessing your patient's behaviour

(B) Just getting stuck in

(C) Not to involve the veterinary surgeon

(D) Obtain a history and perform a clinical examination of the musculoskeletal system

\title{
Which technique for what
}

\section{Gillian Calvo}

\section{INDICATIONS FOR PHYSIOTHERAPY}

Physiotherapy may be indicated in a variety of orthopaedic clinical conditions associated with the musculoskeletal system. Physiotherapy can be used in conjunction with a postoperative treatment plan such as a cranial cruciate ligament repair and may also be implemented for patients undergoing conservative management of a disease process such as osteoarthritis or degenerative myelopathy. To maximize the benefits, and the patient's response to physiotherapy, treatment should be started as soon as possible unless contraindicated

\section{AIM OF PHYSIOTHERAPY}

A range of physiotherapeutic modalities are used in combination to restore maximum functional mobility. Physiotherapy is used to:

- Reduce pain

- Reduce inflammation, swelling

- Promote healing of musculoskeletal tissues

- Improve strength
- Improve core strength and stability

- Improve joint flexibility

- Restore functional movement

- Improve cardiovascular fitness

- Increase speed of recovery

Modalities, indications, effects, contraindications and therapy options as outlined below will be discussed in relation to the orthopaedic patient during the lecture. These will include:

- Thermotherapy

- Cold and heat therapy

- Massage therapy

- Effleurage

- Petrissage

- Friction

- Shaking

- Percussion

\section{THERAPEUTIC EXERCISES}

Therapeutic exercises are an important aspect of patient rehabilitation to improve the rate of recovery and the quality of movement achievable by the patient including improved stamina and cardiovascular fitness. Patient rehabilitation plans should not be stagnant but should evolve depending on progress. It is important to remember that if your patient is not fulfilling the set goals or achieving the best performance from the exercises prescribed then perhaps the modality should be changed 


\section{Orthopaedics and physiotherapy (VN)}

or maybe you are challenging them sooner than they are capable of delivering. Evaluate your patient's performance on a daily basis and amend the rehabilitation plan accordingly.

\section{Goals of therapeutic exercises}

- Improve muscle mass and strength

- Improve core stability and balance

- Improve active pain-free range of motion in the joints and limbs overall

- Improve flexibility

- Reduce lameness

- Improve limb use

- Improve ability to perform activities of daily living

- Prevent further injury though owner education

\section{Assisted therapeutic exercises}

Assisted therapeutic exercises comprise a group of exercises that revolve around standing and can be used to help patients that have some ability to bear weight but are too weak. Various support devices are available that provide more or less support depending on the strength and weight-bearing ability of the patient.

Examples of therapeutic exercises include:

- Assisted standing exercises

- Weight-shifting

- Downward pelvic/shoulder pressure

- Side bending

- Cervical flexion and extension

- Balancing/wobble boards

\section{Active therapeutic exercises}

As suggested, active therapeutic exercises are beneficial for patients who are able to exercise with very little, or no support. The exercises are used to improve the patient's strength, cardiovascular fitness and overall functional ability.
Examples of active therapeutic exercises include:

- Supported walking

- Sit-to-stand

- Dancing exercises

- Wheel barrowing

- Cavaletti poles

- Weaving poles

\section{KEY LEARNING OBJECTIVES}

- Understand the key indications for physiotherapy

- Be able to convey the key aims of physiotherapy to clients and colleagues

- Understand the various physiotherapy techniques available in patient rehabilitation

\section{MULTIPLE CHOICE QUESTIONS}

1. Which of the following is an indication for physiotherapy?

(A) Postoperative rehabilitation of orthopaedic conditions

(B) Patient bonding

(C) Inflammation of the skin

(D) Improve wellbeing by passing the time

2. Physiotherapy should begin:

(A) After 1 week

(B) After 3 weeks

(C) After 6 weeks

(D) As soon as possible unless contraindicated

3. Which of the following is not an aim of physiotherapy

(A) Restore functional movement

(B) Promote healing of musculoskeletal tissues

(C) Increase speed of recovery

(D) Prolong healing of musculoskeletal tissues

\section{When to use hydrotherapy}

\section{Gillian Calvo}

Hydrotherapy is predominantly used for the management of orthopaedic disorders. It is based on the principles of water properties and how they react with objects within it. During the lecture case studies will be discussed relating hydrotherapy to orthopaedic conditions.

\section{PRINCIPLES OF HYDROTHERAPY}

\section{Patient density}

This determines how well the patient will float on water as it determines the weight of the patient compared with an equal volume of water. The density of water is 1.0 compared with 0.8 for fat and 2.0 for bone; therefore fat will float and bone will sink. The implication of this is that a lean patient will need more support in the water than an obese patient.

\section{Buoyancy}

Describes the upward thrust of water on an immersed or partially immersed (floating) object, equivalent to the weight of water that is displaced by the object. This coincides with the relative density of the patient; i.e. buoyancy creates an apparent loss of weight of the patient while in the water. If gravity is not over the centre of the patient's body on the vertical or horizontal plane then the patient is likely to roll or be nose up/bottom down in the water or vice versa. This has practical importance if using floatation devices around the midriff or pelvic region of the patient. Immersion depth can be adjusted in accordance with the clinical condition of the patient and the desired effect of the treatment. 